\title{
REPRESENTATIVES OF THE THOM CLASS OF A VECTOR BUNDLE ${ }^{\dagger}$
}

\author{
Michel Bauer ${ }^{1}$ and Frank Thuillier ${ }^{2}$ \\ Laboratoire de Physique Théorique ENSLAPP用
}

\begin{abstract}
After a review of several methods designed to produce equivariant cohomology classes, we apply one introduced by Berline, Getzler and Vergne to get a family of representatives of the universal Thom class of a vector bundle. Surprisingly, this family does not contain the representative given by Mathaï and Quillen. However it contains the particularly simple and symmetric representative of Harvey and Lawson.
\end{abstract}

*URA 14-36 du CNRS, associée à l'Ecole Normale Supérieure de Lyon, $\dagger$ Work partially supported by European Community Contract ERBCHRXCT920069

1 Groupe d'Annecy: LAPP, Chemin de Bellevue BP 110, F-74941 Annecy-le-Vieux Cedex, France.

${ }^{2}$ Groupe de Lyon: ENS Lyon, 46 allée d'Italie, F-69364 Lyon Cedex 07,France. 


\section{Introduction.}

In a recent paper [STW94] it has been shown how equivariant cohomology is related to the so-called (cohomological) topological models B192, BS88, BS91, OSB89, W88, WBS88. In the same work, a way to compute some representatives of equivariant cohomology classes (i.e. observables of the corresponding topological model) was exhibited.

Here, we shall use this method in order to generate a family of representatives of the Thom class of a vector bundle depending on two arbitrary functions. As we shall see, these representatives are quite different from the Mathaï-Quillen representative. They offer a good deal of flexibility at the price of being slightly complicated. Special choices allow to find a very special representative with remarkable symmetry properties. However, its slow decrease at infinity makes it necessary to consider a cohomology theory with coefficients with sufficiently fast decrease (instead of compact). Some of these representatives (in particular the most symmetric one) already appeared in a quite different framework in the work of Harvey and Lawson on singular connections [HL93], a fact we learned after this work was completed.

This work is divided into three parts. In the first section we recall basic facts about equivariant cohomology as well as the way to compute representatives of equivariant cohomology classes. This section parallels the explanations given in [STW94]. The second section is devoted to the Mathai--Quillen representative of the Thom class. Finally, the last section exhibits a large family of representatives of the Thom class.

\section{Equivariant cohomology.}

Let us consider the following setting : $\mathcal{M}$ is a smooth manifold and $\mathcal{G}$ a connected Lie group acting smoothly on $\mathcal{M}$. We would like to define a cohomology of the quotient space $\mathcal{M} / \mathcal{G}$ which coincides with the De Rham cohomology when this quotient is a smooth manifold but which also exists when it is not, i.e. when $\mathcal{G}$ acts with fixed points. Equivariant cohomology solves this problem.

Let $\mathcal{M}$ be a smooth manifold and $\Omega^{*}(\mathcal{M})$ the exterior algebra of differential forms on $\mathcal{M}$ endowed with the differential $d_{\mathcal{M}}$. A Lie group $\mathcal{G}$ is assumed to be acting on $\mathcal{M}$ as well as its Lie algebra, denoted $\operatorname{Lie} \mathcal{G}$. For any $\lambda \in$ Lie $\mathcal{G}$ there is a vector field $\lambda_{\mathcal{M}}$ representing the infinitesimal action of $\lambda$ on $\mathcal{M}$. This vector field $\lambda_{\mathcal{M}}$ is usually called the fundamental vector field associated with $\lambda$. We shall denote by $i_{\mathcal{M}}(\lambda)=i_{\mathcal{M}}\left(\lambda_{\mathcal{M}}\right)$ and

$l_{\mathcal{M}}(\lambda)=l_{\mathcal{M}}\left(\lambda_{\mathcal{M}}\right)=\left[d_{\mathcal{M}}, i_{\mathcal{M}}(\lambda)\right]_{+}$the contraction (or inner derivative) and Lie derivative acting on $\Omega^{*}(\mathcal{M})$. Let us recall that $i_{\mathcal{M}}(\lambda)$ takes n-forms into (n-1)-forms while $l_{\mathcal{M}}(\lambda)$ acts on forms without changing the degree. Elements of $\Omega^{*}(\mathcal{M})$ which are annihilated by both $i_{\mathcal{M}}(\lambda)$ and $l_{\mathcal{M}}(\lambda)$, for any $\lambda \in$ Lie $\mathcal{G}$, are the so-called basic elements of $\Omega^{*}(\mathcal{M})$ 
for the action of $\mathcal{G}$. As $d_{\mathcal{M}}$ maps basic elements into basic elements, this leads to the definition of the basic cohomology of $\mathcal{M}$ for the action of $\mathcal{G}$ [C50].

We now consider the Weil algebra $\mathcal{W}(\mathcal{G})$ of Lie $\mathcal{G}$. It is a graded differential algebra generated by two $\operatorname{Lie} \mathcal{G}$-valued indeterminates, the "connection" $\omega$, of degree 1, and its "curvature" $\Omega$, of degree 2 , such that :

$$
\Omega=d_{\mathcal{W}} \omega+\frac{1}{2}[\omega, \omega]
$$

where $d_{\mathcal{W}}$ is the differential of $\mathcal{W}(\mathcal{G})$. Of course, one has the Bianchi identity :

$$
d_{\mathcal{W}} \Omega+[\omega, \Omega]=0
$$

There is an action $i_{\mathcal{W}}(\lambda), l_{\mathcal{W}}(\lambda)$ for $\lambda \in$ Lie $\mathcal{G}$ :

$$
\begin{array}{lll}
i_{\mathcal{W}}(\lambda) \omega=\lambda & & l_{\mathcal{W}}(\lambda) \omega=-[\lambda, \omega] \\
i_{\mathcal{W}}(\lambda) \Omega=0 & & l_{\mathcal{W}}(\lambda) \Omega=-[\lambda, \Omega]
\end{array}
$$

For instance, $\omega$ may be a connection on a principal $\mathcal{G}$-bundle $\Pi$ and $\Omega$ its curvature. In that case $i_{\mathcal{W}}(\lambda)$ and $l_{\mathcal{W}}(\lambda)$ are generated by the action of $\mathcal{G}$ on $\Pi$, and in this case $\mathcal{W}(\mathcal{G})$ will be referred to as $\mathcal{W}_{\Pi}$.

We now consider the graded differential algebra $\left(\Omega^{*}(\mathcal{M}) \otimes \mathcal{W}(\mathcal{G}), d_{\mathcal{M}}+d_{\mathcal{W}}\right)$, on which the operations $i_{\mathcal{M}}+i_{\mathcal{W}}$ and $l_{\mathcal{M}}+l_{\mathcal{W}}(\lambda)$ for any $\lambda \in L i e \mathcal{G}$ are well-defined. There common kernel is a graded differential subalgebra of $\Omega^{*}(\mathcal{M}) \otimes \mathcal{W}(\mathcal{G})$. By definition, the so-called equivariant cochains are the elements of this subalgebra annihilated by the differential $d_{\mathcal{M}}+d_{\mathcal{W}}$, leading to the equivariant cohomology of $\mathcal{M}$ for the action of $\mathcal{G}$ : this is the so-called Weil model for equivariant cohomology.

Equivariant cohomology can be alternatively described in the so-called intermediate model, which was introduced in [K93] and which will be repeatedly used in the sequel. It is obtained from the Weil model via of the following algebra isomorphism $\mathrm{y}^{3}$ :

$$
x \longmapsto \exp \left\{-i_{\mathcal{M}}(\lambda)\right\} x
$$

for any $x \in \Omega^{*}(\mathcal{M}) \otimes \mathcal{W}(\mathcal{G})$. This isomorphism changes the original differential and operations on $\Omega^{*}(\mathcal{M}) \otimes \mathcal{W}(\mathcal{G})$ by conjugation :

$$
\begin{aligned}
d_{\mathcal{M}}+d_{\mathcal{W}} & \left.\longrightarrow D_{i n t}=d_{\mathcal{M}}+d_{\mathcal{W}}+l_{\mathcal{M}}(\omega)-i_{\mathcal{M}}(\Omega)\right) \\
\left(i_{\mathcal{M}}+i_{\mathcal{W}}\right)(\lambda) & \longrightarrow i_{\mathcal{W}}(\lambda)=e^{-i_{\mathcal{M}}(\lambda)}\left(i_{\mathcal{M}}+i_{\mathcal{W}}\right)(\lambda) e^{i_{\mathcal{M}}(\lambda)} \\
\left(l_{\mathcal{M}}+l_{\mathcal{W}}\right)(\lambda) & \longrightarrow\left(l_{\mathcal{M}}+l_{\mathcal{W}}\right)(\lambda)=e^{-i_{\mathcal{M}}(\lambda)}\left(l_{\mathcal{M}}+l_{\mathcal{W}}\right)(\lambda) e^{i_{\mathcal{M}}(\lambda)}
\end{aligned}
$$

\footnotetext{
${ }^{2}$ This is a harmless abuse of notation, but it is to be remembered that equivariant cohomology deals only with the local structure of $\mathcal{G}$.

${ }^{3}$ See DV93 for a more general theorem.
} 
Finally, the so-called Cartan model is obtained from the intermediate model by putting $\omega=0$ so that $\left.D_{i n t}^{2}\right|_{\omega=0}$ vanishes when restricted to invariant cochains. This is the most popular model, although many calculations are better automatized in the intermediate model.

Another item which will be repeatedly used is "Cartan's theorem 3" C50 : let us assume that $\left(\Omega^{*}(\mathcal{M}), d_{\mathcal{M}}, i_{\mathcal{M}}, l_{\mathcal{M}}\right)$ admits a $\mathcal{G}$-connection $\theta$, with curvature $\Theta$. Then any equivariant cohomology class of $\Omega^{*}(\mathcal{M}) \otimes \mathcal{W}(\mathcal{G})$ with representative $P(\omega, \Omega)$ gives rise canonically to a basic cohomology class of $\Omega(\mathcal{M})$ with representative $P(\theta, \Theta)$. There is a simple proof using the homotopy that expresses the triviality of the cohomology of the Weil algebra MSZ85]. It follows from the construction that the cohomology class of $P(\theta, \Theta)$ does not depend on $\theta$.

One convenient way to produce equivariant cohomology classes is as follows [BGV91] : we consider an $H$-bundle $\mathcal{P}(\mathcal{M}, H)$ over $\mathrm{M}$ on which there exists an action of $\mathcal{G}$ which lifts the action of $\mathcal{G}$ on $\mathcal{M}$. In general, the Lie group $H$ has nothing to do with the Lie group $\mathcal{G}$. As before, $\mathcal{P}(\mathcal{M}, H)$ is endowed with a differential $d_{\mathcal{P}}$, a contraction $i_{\mathcal{P}}$ and a Lie derivative $l_{\mathcal{P}}$.

Next, let $\Gamma$ be a $\mathcal{G}$-invariant $H$-connection on $\mathcal{P}(\mathcal{M}, H)$ :

$$
l_{\mathcal{P}}(\lambda) \Gamma=0 \quad, \quad \text { for any } \lambda \in \operatorname{Lie} \mathcal{G}
$$

The pull-back $\hat{\Gamma}$ of $\Gamma$ on $\Omega^{*}(\mathcal{M}) \otimes \mathcal{W}(\mathcal{G})$ is a 1 -form on $\mathcal{P}(\mathcal{M}, H)$ and a 0 -form in $\mathcal{W}(\mathcal{G})$. It follows that :

$$
i_{\mathcal{W}}(\lambda) \hat{\Gamma}=0
$$

for any $\lambda \in$ Lie $\mathcal{G}$.

In $\Omega^{*}(\mathcal{M}) \otimes \mathcal{W}(\mathcal{G})$, the equivariant curvature of $\hat{\Gamma}$ is defined by :

$$
R_{\text {int }}^{e q}(\hat{\Gamma}, \omega, \Omega)=D_{\text {int }} \hat{\Gamma}+\frac{1}{2}[\hat{\Gamma}, \hat{\Gamma}]
$$

where $D_{\text {int }}=d_{\mathcal{W}}+d_{\mathcal{P}}+l_{\mathcal{P}}(\omega)-i_{\mathcal{P}}(\Omega)$. Then, if $I_{H}$ is a symmetric invariant polynomial on $\operatorname{Lie} H$, we consider the $H$-characteristic class $I_{H, i n t}^{e q}(\hat{\Gamma}, \omega, \Omega)=I_{H}\left(R_{H, i n t}^{e q}(\hat{\Gamma}, \omega, \Omega)\right)$. It is defined on $\mathcal{M}$ and fulfills :

$$
\begin{aligned}
\left(d_{\mathcal{W}}+d_{\mathcal{M}}+l_{\mathcal{M}}(\omega)-i_{\mathcal{M}}(\Omega)\right) I_{H, i n t}^{e q}(\hat{\Gamma}, \omega, \Omega) & =0 \\
i_{\mathcal{W}}(\lambda) I_{H, i n t}^{e q}(\hat{\Gamma}, \omega, \Omega) & =0 \\
\left(l_{\mathcal{W}}+l_{\mathcal{M}}\right)(\lambda) I_{H, i n t}^{e q}(\hat{\Gamma}, \omega, \Omega) & =0
\end{aligned}
$$

for any $\lambda \in$ Lie $\mathcal{G}$.

\footnotetext{
${ }^{4}$ that is to say a $\operatorname{Lie} \mathcal{G}$-valued 1 -form on $\mathcal{M}$ such that $i_{\mathcal{M}}(\lambda) \theta=\lambda$ and $l_{\mathcal{M}}(\lambda) \theta=-[\lambda, \theta]$ for any $\lambda \in \operatorname{Lie} \mathcal{G}$.
} 
In the Weil model, the equivariant curvature is defined by :

$$
R_{W}^{e q}(\hat{\Gamma}, \omega, \Omega)=\left(d_{\mathcal{W}}+d_{\mathcal{P}}\right) \hat{\Gamma}+\frac{1}{2}\left[\hat{\Gamma}+i_{\mathcal{P}}(\Omega) \hat{\Gamma}, \hat{\Gamma}+i_{\mathcal{P}}(\Omega) \hat{\Gamma}\right]
$$

We may similarly consider :

$$
I_{H, W}^{e q}(\hat{\Gamma}, \omega, \Omega)=I_{H}\left(R_{H, W}^{e q}(\hat{\Gamma}, \omega, \Omega)\right)=e^{-i \mathcal{M}(\lambda)} I_{H, i n t}^{e q}(\hat{\Gamma}, \omega, \Omega)
$$

which fulfills :

$$
\begin{aligned}
\left(d_{\mathcal{W}}+d_{\mathcal{M}}\right) I_{H, W}^{e q}(\hat{\Gamma}, \omega, \Omega) & =0 \\
\left(i_{\mathcal{W}}+i_{\mathcal{M}}\right)(\lambda) I_{H, W}^{e q}(\hat{\Gamma}, \omega, \Omega) & =0 \\
\left(l_{\mathcal{W}}+l_{\mathcal{M}}\right)(\lambda) I_{H, W}^{e q}(\hat{\Gamma}, \omega, \Omega) & =0
\end{aligned}
$$

for any $\lambda \in$ LieG.

Finally, if $\mathcal{M}$ admits a $\mathcal{G}$-connection $\theta$ with curvature $\Theta$, we can apply "Cartan's theorem 3", and substitute $\theta$ and $\Theta$ instead of $\omega$ and $\Omega$ in $I_{H, W}^{e q}(\hat{\Gamma}, \omega, \Omega)$, so that :

$$
\begin{array}{r}
d_{\mathcal{M}} I_{H, W}^{e q}(\hat{\Gamma}, \theta, \Theta)=0 \\
i_{\mathcal{M}}(\lambda) I_{H, W}^{e q}(\hat{\Gamma}, \theta, \Theta)=0 \\
l_{\mathcal{M}}(\lambda) I_{H, W}^{e q}(\hat{\Gamma}, \theta, \Theta)=0
\end{array}
$$

for any $\lambda \in$ LieG.

By standard arguments, these cohomology classes do not depend either on $\hat{\Gamma}$ or on $\theta$.

\section{The Thom Class of a Vector Bundles : the Mathaï- Quillen strategy [MQ86].}

Let $V$ be a real oriented Euclidean vector space of dimension $n=2 d$ with scalar product $(,)_{V}$. On $V$, we choose a canonical basis $\left\{\vec{e}_{k}\right\}$ orthonormal with respect to $(,)_{V}$ :

$$
\left(\vec{e}_{i}, \vec{e}_{j}\right)_{V}=\delta_{i j}
$$

Any vector on $V$ can be decomposed as :

$$
\vec{v}=v^{k} \vec{e}_{k}
$$

Such a decomposition gives a coordinates system $\left(v^{k}\right)$ on $V$, turning $V$ into a manifold. Due to the linear space structure of $V$, only $G L(n, \mathbb{R})$ transformations define allowed 
coordinate changes. The group of isometries of $V$, with respect to $(,)_{V}$, is $S O(n) \subset$ $G L(n, \mathbb{R})$, with Lie algebra $s o(n)$ and Weil algebra $\mathcal{W}(S O(n))$. Finally, we endow $V$ and $\mathcal{W}(S O(n))$ with the standard differential operations $d_{V}, i_{V}, l_{V}, d_{\mathcal{W}}, i_{\mathcal{W}}$ and $l_{\mathcal{W}}$.

Now, let $E(\mathcal{M}, V)$ be a vector bundle over a smooth manifold $\mathcal{M}$ with typical fiber $V$, equipped with differential operations : $d_{E}, i_{E}$ and $l_{E}$, . We denote $\Omega_{r d v}^{n}(E)$ the space of nforms on $E$ whose restriction to each fiber of $E$ is rapidly decreasing. The corresponding cohomology space is written $H_{r d v}^{n}(E)$. The Thom Class of $E$ is the element $\mathrm{T}(E)$ of $H_{r d v}^{n}(E)$ such that :

$$
\int_{V} T(E)=1
$$

which means that integration of $\mathrm{T}(E)$ along the fiber produces the constant function 1 on $\mathcal{M}$.

Actually, following Mathaï and Quillen [MQ86], we would like to exhibit a representative of $\mathrm{T}(E)$ in the form of an integral representation. Then, we consider $V^{*}$, the dual space of $V$, equipped with the scalar product $(,)_{V^{*}}$, dual to $(,)_{V}$ on $V$. Moreover, we introduce coordinates $\left(\varpi_{k}\right)$ for the Grassmann algebra $\Lambda V^{*}$ of $V^{*}$ together with the differential operations $\delta, I$ and $L$, dual to those on $V$.

We take as structure equations :

$$
\begin{aligned}
s^{t o p} v^{k} & =\Psi^{k}+L^{t o p}(\omega) v^{k} \\
s^{t o p} \Psi^{k} & =-L^{t o p}(\Omega) v^{k}+L^{t o p}(\omega) \Psi^{k} \\
s^{t o p} \varpi_{k} & =b_{k}+L^{t o p}(\omega) \varpi_{k} \\
s^{t o p} b_{k} & =-L^{t o p}(\Omega) \varpi_{k}+L^{t o p}(\omega) b_{k} \\
s^{t o p} \omega & =\Omega-\frac{1}{2}[\omega, \omega] \\
s^{t o p} \Omega & =-[\omega, \Omega]
\end{aligned}
$$

with :

$$
\begin{aligned}
s^{\text {top }} & =d_{\mathcal{W}}+\left(d_{V}+\delta\right)+\left(l_{V}+L\right)(\omega)-\left(i_{V}+I\right)(\Omega) \\
\Psi^{k} & =d_{V} v^{k} \equiv \Psi_{i n t}^{k}
\end{aligned}
$$

in the intermediate model, and :

$$
\begin{aligned}
s^{\text {top }} & =d_{\mathcal{W}}+d_{V}+\delta \\
\Psi^{k} & =\left(d_{V}-L^{\text {top }}(\omega)\right) v^{k} \equiv \Psi_{W}^{k}
\end{aligned}
$$

in the Weil model, while :

$$
L^{\text {top }}=l_{V}+L
$$

in any model. 
The null section $s_{0}$ of $E(\mathcal{M}, V)$ that sends any point of $\mathcal{M}$ into the null vector, diffeomorphically maps $\mathcal{M}$ into $s_{0}(\mathcal{M}) \subset E$. Then, the Thom Class $\mathrm{T}(E)$ of $E$ is nothing but the Poincaré dual of $s_{0}(\mathcal{M})$ in $E$ [BT82], and the Dirac form on $E$ :

$$
\delta(\vec{v}) d v^{1} \wedge \ldots \wedge d v^{n}
$$

represents the Poincaré dual of $s_{0}(\mathcal{M})$ in $E$. This form can be written as a Fourier transform :

$$
\frac{1}{(2 \pi)^{n}} \int d b d \varpi \exp i\{b \cdot \vec{v}+\varpi \cdot \Psi\}=\frac{1}{(2 \pi)^{n}} \int d b d \varpi \exp i\left\{b_{k} v^{k}+\varpi_{k} \Psi^{k}\right\}
$$

From the structure equations (26), we deduce :

$$
b \cdot \vec{v}+\varpi \cdot \Psi=s^{t o p}(\varpi \cdot \vec{v})
$$

However, we can consider a smoother representative, with a gaussian behavior for instance. That means that we must insert a term of the form :

$$
i(b, b)_{V^{*}}
$$

into (34). Now, we can try to write the new argument as a $s^{\text {top }}$-exact term :

$$
s^{t o p}\left(\varpi \cdot \vec{v}+i(\varpi, b)_{V_{*}}\right)=b \cdot \vec{v}+\varpi \cdot \Psi+i(b, b)_{V^{*}}-i\left(L^{t o p}(\Omega) \varpi, \varpi\right)_{V^{*}}
$$

so that we are led to define :

$$
U=\frac{1}{(2 \pi)^{n}} \int d b d \varpi \exp i\left\{s^{t o p}\left(\varpi \cdot \vec{v}+i(\varpi, b)_{V_{*}}\right)\right\}
$$

Note that $\mathrm{U}$ is an element of $\mathcal{W}(S O(n)) \otimes \Omega^{*}(V)$.

In order to prove that $\mathrm{U}$ maps into a representative of $\mathrm{T}(E)$, let us proceed in the intermediate model where we write $U_{\text {int }}$ instead of $U$. Then, since in (37) $\omega \in \mathcal{W}(S O(n))$ does not appear, we immediately conclude that $U_{\text {int }}$ does not explicitly depend on $\omega$, that is to say :

$$
\forall \lambda \in \operatorname{so}(n) \quad i_{\mathcal{W}}(\lambda) U_{\text {int }}=0
$$

which express the basicity condition within the intermediate model. Now, there remains to show that $U_{\text {int }}$ is closed with respect to $D_{\text {int }}=d_{\mathcal{W}}+d_{V}+l_{V}(\omega)-i_{V}(\Omega)$. Indeed :

$$
\begin{aligned}
D_{\text {int }} U_{\text {int }} & =\frac{1}{(2 \pi)^{n}} D_{\text {int }} \int d b d \varpi \exp \left\{i \cdot s^{t o p}\left(\varpi \cdot \vec{v}+i(\varpi, b)_{V_{*}}\right)\right\} \\
& =\frac{1}{(2 \pi)^{n}} \int d b d \varpi\left(s^{t o p}-D_{V^{*}}\right) \exp \left\{i \cdot s^{t o p}\left(\varpi \cdot \vec{v}+i(\varpi, b)_{V_{*}}\right)\right\}
\end{aligned}
$$


where $D_{V^{*}}=\delta+L(\omega)-I(\Omega)$. Hence :

$$
D_{i n t} U_{i n t}=-\frac{1}{(2 \pi)^{n}} \int d b d \varpi\left[D_{V^{*}} \exp \left\{i . s^{t o p}\left(\varpi \cdot \vec{v}+i(\varpi, b)_{V_{*}}\right)\right\}\right]
$$

Now, from the structure equations (26), we get :

$$
\begin{aligned}
D_{V^{*}} & =\left(b_{k}+L^{t o p}(\omega) \varpi_{k}\right) \frac{\partial}{\partial \varpi_{k}}+\left(-L^{t o p}(\Omega) \varpi_{k}+L^{t o p}(\omega) b_{k}\right) \frac{\partial}{\partial b_{k}} \\
& =\left(L^{t o p}(\omega) \varpi_{k} \frac{\partial}{\partial \varpi_{k}}+L^{t o p}(\omega) b_{k} \frac{\partial}{\partial b_{k}}\right)+\left(b_{k} \frac{\partial}{\partial \varpi_{k}}-L^{t o p}(\Omega) \varpi_{k} \frac{\partial}{\partial b_{k}}\right)
\end{aligned}
$$

The first term in $D_{V^{*}}$ corresponds to an $s o(n)$-transformation. Due to the $s o(n)$-invariance of the measure $d b d \varpi$, it does not contribute to (41). The last term in (43) vanishes upon integration by parts. Then :

$$
D_{\text {int }} U_{\text {int }}=0
$$

Finally, combining equations (38) and (44), we deduce that:

$$
\forall \lambda \in \operatorname{so}(n) \quad\left(l_{\mathcal{W}}+l_{V}\right)(\lambda) U_{\text {int }}=0
$$

and conclude that $U_{\text {int }}$ is a representative in $\mathcal{W}(S O(n)) \otimes \Omega^{*}(V)$ of the Thom Class of $E(\mathcal{M}, V)$. The corresponding representative in the Weil model is obtained by setting :

$$
\begin{aligned}
s^{t o p} & =d_{\mathcal{W}}+d_{V}+\delta \\
\Psi^{k} & =\left(d_{V}-L^{t o p}(\omega)\right) v^{k} \equiv \Psi_{W}^{k}
\end{aligned}
$$

within equation (37).

Actually, it can be easily shown that Fourier transform (denoted $\mathcal{F}$ ) commutes with equivariant differential operations. More precisely :

$$
\begin{aligned}
\mathcal{F}\left[\left(d_{\mathcal{W}}+\delta+L(\omega)-I(\Omega)\right) \Phi\right] & =\left(d_{\mathcal{W}}+d_{V}+l_{V}(\omega)-i_{V}(\Omega)\right) \mathcal{F}[\Phi] \\
\mathcal{F}\left[i_{\mathcal{W}}(\lambda) \Phi\right] & =i_{\mathcal{W}}(\lambda) \mathcal{F}[\Phi] \\
\mathcal{F}\left[\left(l_{\mathcal{W}}+L\right)(\lambda) \Phi\right] & =\left(l_{\mathcal{W}}+l_{V}\right)(\lambda) \mathcal{F}[\Phi]
\end{aligned}
$$

in the intermediate model. The same holds in the Weil model with suitable differentials. Let us point out that this mainly relies on the identity $b \cdot \vec{v}+\varpi \cdot \Psi=s^{t o p}(\varpi \cdot \vec{v})$.

Then, since $\phi=(b, b)_{V^{*}}+\left(L^{t o p}(\Omega) \varpi, \varpi\right)_{V^{*}}$ is equivariant, it is straightforward to find that its Fourier transform is also equivariant. This simple remark allows to construct representatives of equivariant cohomology classes using Fourier transform of functions of $\phi$. 
Finally, we can consider a principal $S O(n)$-bundle $P$ over $\mathcal{M}$. It is well known that $P \times{ }_{S O(n)} V$ is a vector bundle isomorphic to $E$, and $P \times V$ is called the principal $S O(n)$ bundle associated with $E(\mathcal{M}, V)$. Hence, as an n-form on $E$, any representative of the Thom Class $\mathrm{T}(E)$ of $E$ comes from a closed $S O(n)$-basic $n$-form on the associated bundle $P \times V$ of $E$. In order to produce such a representative of T $(E)$, we use Cartan's Theorem 3 , that is to say we replace $(\omega, \Omega)$ (in the representative $U$ ) by $(\theta, \Theta$ ), a connection and its curvature on $P(\mathcal{M}, S O(n))$.

\section{Construction of Representatives of Thom Class of Vector Bundles : the Berline-Getzler-Vergne strategy [BGV91].}

In this section, we shall use the strategy explained in section 2) in order to produce representatives of $\mathrm{T}(E)$.

To begin with, we are going to turn $V$ into a Riemannian manifold, i.e. a manifold $V$ with a metric. The tangent bundle of $V$, denoted by $T V$, is obviously isomorphic to $V \times V$. The only $S O(n)$-invariants formed with $\vec{v}$ and $d \vec{v}$ are the three scalar products, so that the general $S O(n)$-invariant metric on $V$ is :

$$
d s^{2}(\vec{v})=e^{\varphi}\left(\left(d v^{i}\right)^{2}+\sigma\left(v^{i} d v^{i}\right)^{2}\right)
$$

where $\varphi$ and $\sigma$ are smooth functions of $t=(\vec{v}, \vec{v})_{V}$ only. The above expression is positive definite if and only if $1+\sigma(t) t>0$ for $t \geq 0$. One can assume if convenient that the metric is asymptotically flat (i.e. that the curvature vanishes at infinity).

We can consider the principal $G L(n, \mathbb{R})$-bundle associated with $T V$, i.e. the frame bundle $R(V)$ of $V$. It is made of the points $\left(\vec{v}, b_{\vec{v}}\right)$ where $b_{\vec{v}}$ is a frame (i.e. a basis) at $\vec{v}$. Coordinates for $b_{\vec{v}}$ are defined as follows. We denoted by $\left(\partial_{k}\right)$ the natural basis of $T_{\vec{v}} V$

defined by the canonical coordinates $\left(v^{k}\right)$ of $V: \partial_{k}=\frac{\partial}{\partial v^{k}}$. Then, the coordinates of $b_{\vec{v}}$ are the components $b_{k}^{j}$ of the decomposition of $b_{\vec{v}}$ with respect to the natural basis $\left(\partial_{k}\right)$ :

$$
b_{\vec{v} k}=b_{k}^{j} \partial_{j}
$$

with $b_{\vec{v} k}$ the $k$-th frame vector of the frame $b_{\vec{v}}$. The isometry group of $\left(V,(,)_{V}\right)$, namely $S O(n)$, acts both on elements of $V$ and on frames, that is to say on $R(V)$. This goes as follows. For any $\Phi \in S O(n)$ :

$$
\Phi^{k}(\vec{v})=\Phi_{m}^{k} v^{m}
$$

At the infinitesimal level, if we write $\Phi_{m}^{k}=\delta_{m}^{k}+\varphi_{m}^{k}$, we get :

$$
\Phi^{k}(\vec{v})=\left(\delta_{m}^{k}+\varphi_{m}^{k}\right) v^{m}=v^{k}+\varphi_{m}^{k} v^{m}=v^{k}+\xi^{k}
$$


where $\xi^{k}=\varphi_{m}^{k} v^{m}$ defines a vector field on $V$, the so-called fundamental vector field associated with the action of $\varphi \in s o(n)$ :

$$
\xi=\xi^{k} \frac{\partial}{\partial v^{k}}
$$

The natural action of $\Phi \in S O(n)$ on $T_{\vec{v}} V$, is given by the so-called differential of $\Phi$ at $\vec{v}$, $d_{\vec{v}} \Phi: T_{\vec{v}} V \rightarrow T_{\Phi(\vec{v})} V:$

$$
\forall X_{\vec{v}} \in T_{\vec{v}} V \quad, \quad \forall f \in C^{\infty}(V) \quad d_{\vec{v}} \Phi X_{\vec{v}}(f)=X_{\vec{v}}(f \circ \Phi)
$$

Applying this definition to the frame vectors $b_{\vec{v} k}$, one gets :

$$
\tilde{b}_{j}^{i}=b_{j}^{m}\left(\partial_{m} \Phi^{i}(\vec{v})\right)=b_{j}^{m} \Phi_{m}^{i}
$$

where $\tilde{b}_{j}^{i}$ are the coordinates of the transformed frame at $\Phi(\vec{v})$. At the infinitesimal level, for $\varphi \in s o(n)$ :

$$
\tilde{b}_{j}^{i}=b_{j}^{m}\left(\delta_{m}^{i}+\varphi_{m}^{i}\right)=b_{j}^{i}+b_{j}^{m} \varphi_{m}^{i}=b_{j}^{i}+b_{j}^{m} \varphi_{m}^{i}=b_{j}^{i}+\Xi_{j}^{i}
$$

Combining equations (54) and (58), we deduce that the fundamental vector field associated with the action of $\varphi \in s o(n)$ on $R(V)$ reads :

$$
\lambda_{R}=\xi^{k} \frac{\partial}{\partial v^{k}}+\Xi_{q}^{p} \frac{\partial}{\partial b_{q}^{p}}=\varphi_{m}^{k} v^{m} \frac{\partial}{\partial v^{k}}+b_{q}^{m} \varphi_{m}^{p} \frac{\partial}{\partial b_{q}^{p}}
$$

Now, let $P(\mathcal{M}, S O(n))$ be some principal $S O(n)$-bundle over a smooth manifold $\mathcal{M}$. It is well known that there is a vector bundle over $\mathcal{M}$ associated with $P$ for the action of $S O(n)$ on $V$. The group $S O(n)$ acts on the right on $P$ and on the left on $V$. We first define a right-action of $S O(n)$ on $P \times V$ by setting :

$$
(p, \vec{v})^{\Phi}=\left(p . \Phi, \Phi^{-1}(\vec{v})\right)
$$

so that, the fundamental vector field representing the action of $\varphi \in \operatorname{so}(n)$ on $P \times V$ reads :

$$
\lambda_{P \times V}=\lambda_{P}-\xi^{k} \frac{\partial}{\partial v^{k}}=\lambda_{P}-\varphi_{m}^{k} v^{m} \frac{\partial}{\partial v^{k}}
$$

where $\lambda_{P}$ is the fundamental vector field representing the action of $\varphi$ on $P$.

Finally, the action of any $\varphi \in s o(n)$ on the $G L(n, \mathbb{R})$-principal bundle $P \times R(V)$, is given by following fundamental vector field :

$$
\lambda=\lambda_{P}-\lambda_{R}
$$

with $\lambda_{R}$ defined in equation (59). 
In the following, $V, R(V)$ and $P$ are equipped with the following differential operations : $d_{V}, d_{R}, d_{P}, i_{V}, i_{R}, i_{P}, l_{V}, l_{R}$ and $l_{P}$, respectively exterior differentials, inner products and Lie derivatives.

Now, since we are looking for representatives of equivariant cohomology classes, we can mimic the construction made in STW94 in the case of two-dimensional Gravity. We first look for a $G L(n, \mathbb{R})$-connection on $\mathcal{P}(P \times V, G L(n, \mathbb{R}))=P \times R(V)$ invariant under the action of $S O(n)$. If we notice that, by construction, the metric $\mathbf{g}$ on $V$ is $S O(n)$ invariant, we can consider the Levi-Cevita connection ${ }^{L C} \Gamma$ associated with $\mathbf{g}$. Due to the $S O(n)$-invariance of $\mathbf{g},{ }^{L C} \Gamma$ is an $S O(n)$-invariant connection. More precisely, the lift of ${ }^{L C} \Gamma$ into a connection one form $\Gamma$ on $R(V)$ according to :

$$
\Gamma=b^{-1}\left({ }^{L C} \Gamma\right) b+b^{-1} d_{R} b
$$

is invariant under the action of $S O(n)$. The fundamental vector field for the action of so $(n)$ was given before, so that:

$$
\left(i_{\mathcal{P}}(\lambda) \Gamma\right)_{\tau}^{\sigma}=\left(b^{-1}\right)_{\nu}^{\sigma}\left(-{ }^{L C} D_{\mu} \xi^{\nu}\right) b_{\tau}^{\mu}
$$

where $i_{\mathcal{P}}(\lambda)=\left(i_{P}+i_{R}\right)(\lambda)$, and :

$$
l_{\mathcal{P}}(\lambda) \Gamma=0
$$

with $l_{\mathcal{P}}(\lambda)=\left(l_{P}+l_{R}\right)(\lambda)$.

The next step is to consider the Weil algebra $\mathcal{W}(S O(n))$ of $s o(n)$. The relevant formulæ were given in section 2 . We recall that the equivariant curvature of $\Gamma$ in the intermediate model is :

$$
R_{\text {int }}^{e q}(\Gamma, \omega, \Omega)=\left(d_{\mathcal{W}}+d_{\mathcal{P}}+l_{\mathcal{P}}(\omega)-i_{\mathcal{P}}(\Omega)\right) \Gamma+\frac{1}{2}[\Gamma, \Gamma]
$$

while the corresponding curvature in the Weil model is obtained as :

$$
R_{W}^{e q}(\Gamma, \omega, \Omega)=e^{i_{\mathcal{P}}(\omega)} R_{\text {int }}^{e q}(\Gamma, \omega, \Omega)
$$

which gives :

$$
R_{W}^{e q}(\Gamma, \omega, \Omega)=R(\Gamma)+i_{\mathcal{P}}(\omega) R(\Gamma)+\frac{1}{2} i_{\mathcal{P}}(\omega) i_{\mathcal{P}}(\omega) R(\Gamma)-i_{\mathcal{P}}(\Omega) \Gamma
$$

The Weil equivariant Euler class is defined by :

$$
E_{W}^{e q}=\frac{\varepsilon^{\mu_{1} \rho_{1} \ldots \mu_{d} \rho_{d}}}{\sqrt{\mathbf{g}}} g_{\rho_{1} v_{1} \ldots} g_{\rho_{d} v_{d}}\left(R_{W}^{e q}\right)_{\mu_{1}}^{v_{1}} \wedge \ldots \wedge\left(R_{W}^{e q}\right)_{\mu d}^{v_{d}}
$$

which after normalization gives rise to a representative of $\mathrm{T}(E)$ in $P(\mathcal{M}, S O(n)) \times V$. 
It is now time to use the explicit form of the metric to get a formula for the Thom class. Surprisingly, we shall see there is no choice of metric that allows to recover the Mathai-Quillen representative of $\mathrm{T}(E)$. From now on, the computations, if painful, are straightforward. We use the intermediate model so that $d_{V} v^{i} \equiv \Psi^{i}$. As (63) looks formally like a change of coordinates in the fiber, we know that its effect on curvature will be a simple conjugation which disappears completely on the Thom class. So we can forget it in the computation. From (51) we find that the metric is :

$$
g_{i j}=e^{\varphi}\left(\delta_{i j}+\sigma v_{i} v_{j}\right)
$$

Our notations need some comment : we start with global coordinates $v^{i}$ on $V$, so the exponent $i$ is not a tensor component but just a label. The metric is expressed with respect to this particular coordinate system. However it is convenient to deal consistently with formal lower and upper indices in the Einstein summation convention. So we define $v_{i} \equiv v^{i}$ and $\delta_{i j} \equiv \delta^{i j} \equiv \delta_{j}^{i} \equiv \delta_{i}^{j}=1$ if $i=j$ and 0 else. For instance we use the notation $v_{i}$ and $\delta_{i j}$ in $g_{i j}$ and we write $t=v_{i} v^{i}$. This becomes slightly less formal if we restrict the diffeomorphism group of $V$ to linear orthogonal transformations.

A simple computation shows that the inverse metric is

$$
g^{i j}=e^{-\varphi}\left(\delta^{i j}+\tilde{\sigma} v^{i} v^{j}\right)
$$

where $\tilde{\sigma}$ is defined by $(1+t \tilde{\sigma})(1+t \sigma)=1$.

First, we need a formula for the connection and curvature. The fact that $\varphi$ and $\sigma$ depend only on $t$ leads to many simplifications in the computation. We use dots for derivatives with respect to $t$.

With the expression of $\mathbf{g}$, we get for the connection ${ }^{5}$ :

$$
\Gamma_{i j}^{k}=(1+t \tilde{\sigma}) v^{k}\left[(\sigma-\dot{\varphi}) \delta_{i j}+(\dot{\sigma}-\sigma \dot{\varphi}) v_{i} v_{j}\right]+\dot{\varphi}\left(v_{i} \delta_{j}^{k}+v_{j} \delta_{i}^{k}\right) .
$$

so that the connection matrix is

$$
\Gamma_{i}^{j} \equiv \Psi^{k} \Gamma_{i k}^{j}=A v^{j} v_{i} v_{k} \Psi^{k}+B v^{j} \Psi_{i}+C\left(v_{i} \Psi^{j}+\delta_{i}^{j} v_{k} \Psi^{k}\right)
$$

where we have set

$$
\left\{\begin{array}{l}
A=(1+t \tilde{\sigma})(\dot{\sigma}-C \sigma) \\
B=(1+t \tilde{\sigma})(\sigma-C) \\
C=\dot{\varphi}
\end{array} .\right.
$$

The curvature matrix is given by

$$
R_{i}^{j} \equiv d \Gamma_{i}^{j}+\Gamma_{k}^{j} \wedge \Gamma_{i}^{k} .
$$

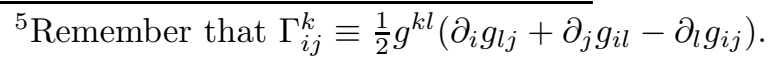


A tedious computation leads to

$$
R^{i j} \equiv g^{i k} R_{k}^{j}=e^{-\varphi}(1+t \tilde{\sigma})\left[M_{11} \Psi^{i} \Psi^{j}+M_{12}\left(v^{i}\left(v_{k} \Psi^{k}\right) \Psi^{j}-v^{j}\left(v_{k} \Psi^{k}\right) \Psi^{i}\right)\right]
$$

where

$$
\left\{\begin{array}{l}
M_{11}=C^{2} t+2 C-\sigma \\
M_{12}=2 \dot{C}-C^{2}-\dot{\sigma}+(1+t \tilde{\sigma})(\sigma-C) \dot{t} \dot{\sigma}
\end{array} .\right.
$$

To get the full equivariant curvature, we need the part involving $\Omega$. In accordance with our convention on indices, we define $\Omega^{i j} \equiv \Omega_{i}^{j}$. By definition $\Omega^{i j}$ is antisymmetric. According to formulæ $(64,66)$, the part of the equivariant curvature containing $\Omega$ is the covariant derivative of $\Omega_{k}^{j} v^{k}$, the $s o(n)$ vector field associated to $\Omega$. Consequently

$$
\left(-i_{\mathcal{P}}(\Omega) \Gamma\right)_{k}^{j}=\Omega_{k}^{j}+\Omega_{m}^{l} v^{m} \Gamma_{l k}^{j} .
$$

The antisymmetry of $\Omega$ leads to further simplifications. The outcome is :

$$
g^{i k}\left(\Omega_{k}^{j}+\Omega_{m}^{l} v^{m} \Gamma_{l k}^{j}\right)=e^{-\varphi}(1+t \tilde{\sigma})\left[M_{21} \Omega^{i j}+M_{22}\left(v^{i} v_{k} \Omega^{k j}-v^{j} v_{k} \Omega^{k i}\right)\right]
$$

where

$$
\left\{\begin{array}{l}
M_{21}=1+t \sigma \\
M_{22}=C-\sigma
\end{array}\right.
$$

We note the striking similarity between the two contributions. If we define a two-bytwo matrix $N^{i j}$ by

$$
N^{i j}=\left(\begin{array}{cc}
\Psi^{i} \Psi^{j} & \Omega^{i j} \\
v^{i}\left(v_{k} \Psi^{k}\right) \Psi^{j}-v^{j}\left(v_{k} \Psi^{k}\right) \Psi^{i} & v^{i} v_{k} \Omega^{k j}-v^{j} v_{k} \Omega^{k i}
\end{array}\right)
$$

the equivariant curvature can be written as a trace :

$$
\left(R_{i n t}^{e q}\right)^{i j}=e^{-\varphi}(1+t \tilde{\sigma}) \operatorname{Tr} M N^{i j}
$$

The equivariant Euler class is

$$
E_{i n t}^{e q}=2^{n / 2} \sqrt{\mathrm{g}} \text { Pfaff }\left(R_{i n t}^{e q}\right)^{i j} .
$$

with the usual definition of the Pfaffian. Note that $\sqrt{\mathbf{g}}=e^{n \varphi / 2}(1+t \sigma)^{1 / 2}$.

This is the explicit formula for the universal Thom class that we were after. It involves two arbitrary functions of $t, \varphi$ and $\sigma$ (with the mild restriction $1+t \sigma>0$ ) which may be localized at will thus so leaving a fair amount of flexibility.

The first comment to make is that apparently the above representative, which is of course $s o(n)$ invariant when $s o(n)$ acts on $V, \Omega$ and $\Psi$ at the same time, is not invariant 
when $s o(n)$ acts only on $V$. To state it more simply, the $V$ dependence of the Thom class is not only through $t$. This is to be contrasted with the Mathaï-Quillen representative.

Let us deal with a special case first. When $n=2$, it is easy to see that

$$
\varepsilon_{i j} N^{i j}=2\left(\begin{array}{cc}
\Psi^{1} \Psi^{2} & \Omega^{12} \\
t \Psi^{1} \Psi^{2} & t \Omega^{12}
\end{array}\right)
$$

so we have some hope to recover the Mathaï-Quillen formula as a special case. After some manipulations one finds

$$
E_{\text {int }}^{e q}=4 \dot{F} \Psi^{1} \Psi^{2}+2 F \Omega^{12}
$$

where

$$
F \equiv \frac{1+t C}{(1+t \sigma)^{1 / 2}}
$$

So the Thom class depends only on one arbitrary function of $t$, namely $F$, which can easily be adjusted to recover the Mathai-Quillen representative. The correct choice is $F=-\frac{1}{(4 \pi)} \exp (-t / 4)$.

When $n>2$ the situation is more complicated. We shall use a trick to see how much the symmetry of the $s o(n)$ action on $V$ is broken.

The first observation is that under a similarity, the Pfaffian has a simple behavior : if $A$ is a square antisymmetric matrix and $S$ an arbitrary square matrix of the same size with transpose $S^{t}, S^{t} A S$ is again antisymmetric and Pfaff $S^{t} A S=\operatorname{Det} S$ Pfaff $A$. The square of this equation just follows from the multiplicative property of the determinant, and the sign is fixed by the case when $S$ is the identity matrix. So if we can find a matrix $S^{i j}$ (independent of $\Psi$ and $\Omega$ ) such that $S^{t} R_{\text {int }}^{e q} S$ simplifies, we shall end with a simpler formula for the Thom class.

Define a symmetric matrix $S(D)$ of parameter $D$ by

$$
S(D)_{j}^{i}=\delta_{j}^{i}+D v^{i} v_{j}
$$

This matrix is easily diagonalized : the vectors orthogonal to $v^{i}$ are left invariant and $v^{i}$ is multiplied by $1+t D$. So

$$
\text { Det } S(D)=1+t D
$$

and

$$
S(D) S(E)=S(D+E+t D E)
$$

Moreover, if $A^{i j}$ is any antisymmetric matrix, 


$$
(S(D) A S(D))^{i j}=A^{i j}+D\left(v^{i} v_{k} A^{k j}-v^{j} v_{k} A^{k i}\right) .
$$

We apply this identity to the four antisymmetric objects building the two-by-two matrix $N^{i j}$ to get

$$
S(D) N S(D)=\bar{S}(D) N
$$

where $\bar{S}(D)$ is the two-by-two matrix

$$
\left(\begin{array}{cc}
1 & D \\
0 & 1+t D
\end{array}\right) .
$$

In equation (91), the left-hand side involves a product of $n \times n$ matrices, and the $2 \times 2$ indices are spectators whereas on the right-hand side the opposite occurs.

So we can write

$$
S(D) R_{\text {int }}^{e q} S(D)=e^{-\varphi}(1+t \tilde{\sigma}) \operatorname{Tr} M(D) N
$$

with $M(D) \equiv M \bar{S}(D)$, and the Thom class is

$$
E_{i n t}^{e q}=(1+t \sigma)^{(1-n) / 2}(1+t D)^{-1} \mathrm{Pfaff}(\operatorname{Tr} M(D) N) .
$$

We can choose $D$ to simplify the expression of $E_{i n t}^{e q}$.

First we take $D=D_{1}$ where $M\left(D_{1}\right)=\left(\begin{array}{cc}* & 0 \\ * & *\end{array}\right)$. This makes it easy to compute the term in $E_{\text {int }}^{e q}$ that does not involve $\Omega$. The outcome is

$$
E_{\text {int }}^{e q}=n !(1+t \sigma)^{(1-n) / 2}\left(1+t \frac{M_{12}}{M_{11}}\right) M_{11}^{n / 2} \Psi^{1} \cdots \Psi^{n}+\text { terms involving } \Omega .
$$

One can check that this is compatible with (85) for $n=2$.

Second, we take $D=D_{2}$ where $M\left(D_{2}\right)=\left(\begin{array}{cc}* & * \\ * & 0\end{array}\right)$. This makes it easy to compute the term in $E_{\text {int }}^{e q}$ that does not involve $\Psi$. The outcome is

$$
E_{\text {int }}^{e q}=2^{n / 2}(1+t \sigma)^{(1-n) / 2}\left(1+t \frac{M_{22}}{M_{21}}\right) M_{21}^{n / 2} \text { Pfaff } \Omega+\text { terms involving } \Psi
$$

a result which is again compatible with (85) for $n=2$.

Those two terms in $E_{\text {int }}^{e q}$ automatically depend only on $t$. On the other hand, the other terms are not scalars for the $s o(n)$ action on $V$. To see this we keep $D=D_{2}$, set $A^{i j}=M_{11} \Psi^{i} \Psi^{j}+M_{21} \Omega^{i j}$ and $B^{i j}=v^{i}\left(v_{k} \Psi^{k}\right) \Psi^{j}-v^{j}\left(v_{k} \Psi^{k}\right) \Psi^{i}$. Using the fact that $x_{k} \Psi^{k}$ squares to 0 we get 


$$
\begin{aligned}
E_{\text {int }}^{e q} & = \\
& (1+t \sigma)^{(1-n) / 2}\left(2^{n / 2}\left(1+t \frac{M_{22}}{M_{21}}\right) \text { Pfaff } A-n / 2 \frac{\text { Det } M}{M_{21}} \varepsilon_{i_{1} j_{1} \cdots i_{n} j_{n}} B^{i_{1} j_{1}} A^{i_{2} j_{2}} \cdots A^{i_{n} j_{n}}\right) .
\end{aligned}
$$

As $\Omega$ and $\Psi$ are independent families of indeterminates, the matrix elements of $A^{i j}$ are independent of each other (except for antisymmetry) and of the matrix elements of $B^{i j}$. So in the expansion of

$$
\varepsilon_{i_{1} j_{1} \cdots i_{n} j_{n}} B^{i_{1} j_{1}} A^{i_{2} j_{2}} \cdots A^{i_{n} j_{n}}
$$

no compensation can occur between $A$-factors and $B$-factors or between different $B$ factors. Moreover $B$-factors contain the full non $s o(n)$ invariant part of the $V$ dependence of the Thom class. So we have the following three possibilities. Either Det $M$ is 0 , or $B^{i j}$ is invariant for the action of $s o(n)$ on $V$, or the representative of the Thom class is not invariant for the action of $s o(n)$ on $V$. The first term of the alternative depends on our choice of $\varphi$ and $\sigma$. The second is easily checked to occur if and only if $n=2$, a case we have already treated.

So finally, we have shown that if $n>2$ the representative of the Thom class is invariant for the $s o(n)$ action on $V$ if and only if Det $M=0$.

We shall now see that despite the fact that apparently our representative of the Thom class depends on two arbitrary functions, the single condition Det $M=0$ fixes it completely. This can be seen as a manifestation of the topological character of the Thom class. We shall also see that the representative we end up with is not the Mathaï-Quillen representative.

From now on, we set Det $M=0$. Explicit computation shows that this equation has a first integral. Namely Det $M=0$ is equivalent to

$$
(1+t C)^{3} \frac{d}{d t}\left(\frac{\sigma}{(1+t C)^{2}}+\frac{1}{t(1+t C)^{2}}-\frac{1}{t}\right)=0 .
$$

The term in parenthesis can be written as

$$
-\frac{C^{2} t+2 C-\sigma}{(1+t C)^{2}} \quad \text { or } \quad \frac{(1+t \sigma)-(1+t C)^{2}}{t(1+t C)^{2}}
$$

Now, we distinguish two cases.

Suppose first that for some value of $t$ the function $1+t C$ vanishes together with its first derivative. Then

$$
M=\frac{1+t \sigma}{t}\left(\begin{array}{cc}
-1 & 1 / t \\
t & -1
\end{array}\right)
$$


As a byproduct, $M_{21}+t M_{22}$ vanishes, and the equivariant Euler class vanishes. So clearly, the function $1+t C$ cannot vanish everywhere if we are to find a non-trivial class. Anyway, the vanishing of $1+t C$ would mean that $e^{\varphi}=t_{0} / t$ for some constant $t_{0}$ leading to a metric singular at the origin. It is likely that in this case, a careful computation with distributions would give a curvature concentrated at the origin, but we are not interested in this anyway.

On the open intervals where $1+t C \neq 0$ the second factor of (99) has to vanish. We get

$$
\frac{\sigma}{(1+t C)^{2}}+\frac{1}{t(1+t C)^{2}}-\frac{1}{t}=\frac{1}{t_{0}}
$$

for some constant $t_{0}$. Using (100), one obtains

$$
M=\frac{1+t C}{t_{0}}\left(\begin{array}{cc}
-(1+t C) & \left(1+\left(t_{0}+t\right) C\right)\left(t_{0}+t\right)^{-1} \\
(1+t C)\left(t_{0}+t\right) & -\left(1+\left(t_{0}+t\right) C\right)
\end{array}\right)
$$

leading to a remarkable simplification of (97) :

$$
E_{i n t}^{e q}=2^{n / 2}\left(\frac{t_{0}}{t_{0}+t}\right)^{1 / 2} \operatorname{Pfaff}\left(\Omega^{i j}-\frac{1}{t_{0}+t} \Psi^{i} \Psi^{j}\right) .
$$

Now, as $M_{21}=1+t \sigma$, which has to remain strictly positive, $1+t C$ cannot vanish at the boundary of an open interval where it is nonzero. This means that $1+C t$ vanishes nowhere, and that formula (104) is valid everywhere. This is our final formula for the equivariant Euler class if we decide to trade flexibility (arbitrary choice of $\varphi$ and $\sigma$ ) for simplicity $(s o(n)$ invariance on $V$, leading to a simple Pfaffian). The Mathaï-Quillen representative never shows up for $n>2$.

Some comments are in order. Usually the Thom class is defined by using function with compact support (differential topology) or rapid decrease at infinity (quantum field theory) on $V$. The Mathaï-Quillen representative belongs to this second category. With the general formula, the freedom on $\varphi$ and $\sigma$ allows us to impose any behavior at infinity 5. On the other hand our rigid proposal for the Thom class does not decrease fast at infinity. Despite the fact that this may be inconvenient in certain applications, we would like to point that it makes sense nevertheless. To define the Thom class, the crucial point is that the cohomology of $V$ with coefficients having compact support or rapid decrease at infinity is concentrated in the dimension of $V$ and one dimensional there. It seems clear that a cohomology of $V$ can be build such as to retain this property and accept our rigid representative as a well-defined cohomology class. For instance $k$-forms on $V$ such that for any non-negative integer $l$ the partial derivatives of order $l$ of the coefficients

\footnotetext{
${ }^{6}$ In fact to localize the Thom class on arbitrary spherical shells if this proves useful.
} 
exist and are $O\left(t^{-\frac{k+l+1}{2}}\right)$ at infinity, endowed with the usual exterior derivative, should work.

In particular, we can normalize things in such a way that the integral on $V$ of the term independent of $\Omega$ is 1 , as is usual for the Thom class. A simple calculation gives for the normalized Thom class

$$
T_{V}=\frac{1}{(2 \pi)^{d}}\left(\frac{t_{0}}{t_{0}+t}\right)^{1 / 2} \operatorname{Pfaff}\left(\frac{1}{t_{0}+t} \Psi^{i} \Psi^{j}-\Omega^{i j}\right) .
$$

Playing with the value of $t_{0}$ allows to localize around the zero section. This formula already appears in [HL93] as a specialization of another formula for the Thom class.

\section{Conclusion.}

In these notes, we have obtained formulæ for the universal Thom class of a vector bundle. A special choice leads to a rigid representative involving Cauchy-type kernels. It would be very interesting to know whether the Mathaï-Quillen representative, with its Gaussiantype kernel, is also a rigid member of some natural family of representatives.

\section{Acknowledgments}

We are very grateful to Raymond Stora and Georges Girardi for many fruitful discussions. We thank Reese Harvey for making us aware of ref. [HL93, which contains material closely related to this work.

\section{References}

[B192] M. Blau, "The Mathaï Quillen formalism and topological field theory", Lectures at the 28th Karpacz Winter School on Infinite dimensional geometry in physics, Karpacz, Poland, Feb. 1992, NIKHEF-H/92-07, 17-29

[BGV91] N. Berline, E. Getzler and M. Vergne, "Heat Kernels and Dirac Operators", Grundlehren des Mathematischen Wissenschasft 298, Springer-Verlag Berlin Heidelberg 1992

[BS88] L. Baulieu and I.M. Singer, Nucl. Phys. B Proc Supl 15B (1988) 12

[BS91] L. Baulieu and I.M. Singer, Commun. Math. Phys. 135 (1991) 253

[BT82] R. Bott and L.W. Tu, Differential Forms in Algebraic Topology, Springer-Verlag 1982 
[C50] H. Cartan, Colloque de Topologie (Espaces Fibrés), Brussels 1950, CBRM, 15-56

[DV93] M. Duflo and M. Vergne, Sur la cohomologie équivariante des variétés différentiables, Astérisque 215, 1993, 5-108

[HL93] R. Harvey and B. Lawson, A theory of characteristic currents associated with a singular connection", Astérisque 213 (1993), 1-268.

[K93] J. Kalkman, Commun. Math. Phys. 153 (1993) 447

[MQ86] V. Mathaï and D. Quillen, Topology 25 (1986) 85

[MSZ85] J. Manes, R. Stora and B. Zumino, Commun. Math. Phys. 102 (1985) 157

[OSB89] S. Ouvry, R Stora and P. van Baal, Phys. Lett. B220 (1989) 590

[STW94] R Stora, F. Thuillier and J.C. Wallet, Lectures at the 1st Caribbean Spring School of Mathematics and Theoretical Physics, Saint François, Guadeloupe, May 30 - June 13 1993, Proceedings 1995 (preprint ENSLAPP-A-481/94).

[W88] E. Witten, Commun. Math. Phys. 117 (1988) 353

[WBS88] E. Witten, Commun. Math. Phys. 118 (1988) 411. L. Baulieu and I.M. Singer, Commun. Math. Phys. 125 (1989) 227 